\title{
LAS ROMERÍAS, OPORTUNIDAD TURÍSTICA Y RELACIONES SOCIALES ENTRE LOCALES Y VISITANTES. EL CASO DE LA CERDANYA EN CATALUÑNA ${ }^{1}$
}

\author{
José Ma Prat Forga* \\ Universidad Nacional de Educación a Distancia. UNED \\ Gemma Cànoves Valiente** \\ Universidad Autónoma de Barcelona
}

\section{RESUMEN}

En los estos últimos años se están desarrollando nuevas actividades alrededor del turismo cultural-religioso con el objetivo de dar a conocer y mantener vivo el folklore propio de las generaciones pasadas. Entre estos fenómenos histórico-culturales y turísticos están las romerías. Se trata de excursiones grupales, generalmente a pie, a ermitas y santuarios donde, además de las actividades puramente religiosas, también se aprovecha para realizar otros actos festivos vinculados con las tradiciones del lugar. De este modo, con las romerías, algunos lugares religiosos en los territorios de interior se han convertido en puntos de encuentro entre los turistas y la población local, con una triple función: revalorizar el patrimonio local, atraer visitantes y ser lugar de encuentro entre la comunidad local y los turistas. En este trabajo, a partir de documentación y estadísticas sobre el tema y de entrevistas a participantes, se ha analizado el caso de las romerías en la Cerdanya española (los llamados "aplecs"), y se ha confirmado que se trata de unos eventos populares que

Recibido: 3 de marzo de 2017

Devuelto para su revisión: 19 de julio de 2017

Aceptado: 4 de septiembre de 2017

Facultad de Geografía e Historia. Universidad Nacional de Educación a Distancia. Senda del Rey 7. 28040 MADRID. E-mail: jmpratf@gmail.com

** Departamento de Geografía. Universidad Autónoma de Barcelona. Edificio B. 08193 Bellaterra BARCELONA (España).E-mail: gemma.canoves@uab.cat

1 Este artículo se enmarca en el proyecto Turismo de Interior en España: Retos, valorización y Estrategias ante situaciones cambiantes y de crisis para impulsar productos y destinos turísticos, financiado por el Plan Estatal de Investigación Científica y Técnica, del Ministerio de Economía, Industria y Competitividad, con referencia CSO2016-74861-R 
permiten revalorizar turísticamente el territorio, ayudando a reafirmar la identidad local, conservando el patrimonio material e inmaterial y favoreciendo las relaciones sociales entre gente con diferentes intereses y motivaciones.

Palabras clave: romerías, turismo, cultura, historia, desarrollo local, Cerdanya.

The romeries: tourist opportunity and social relationships between local and visitors. the case of la Cerdanya in Catalonia (Spain)

\begin{abstract}
In the latter years new activities are developing about the cultural and religious tourism with the aim to announce and keep alive the own folklore of the past generations. Between these historical, cultural and tourist phenomena this is the pilgrimage. There are group excursions, generally afoot, to hermitages and sanctuaries where, besides the purely religious activities, also he takes advantage to realize other festive acts linked with the local traditions. Thus, with the pilgrimages, some religious places in the territories of interior have turned into points of meeting between the tourists and the local population, with a triple function: to revalue the local heritage, to attract visitors and to be a place of meeting between the local community and tourists. In this work, from documentation and statistics on the topic and of you interview participants, the case of the pilgrimages has been analyzed in the Spanish Cerdanya (the so called "aplecs"), and has been confirmed that treats itself about a few popular events that they allow to revalue the tourism in the territory, reaffirming the local identity, preserving the local heritage and favoring the social relations between people with different interests and motivations.
\end{abstract}

Keywords: Pilgrimages, tourism, culture, history, local development, Cerdanya.

\title{
1. INTRODUCCIÓN
}

Recientemente constatamos que muchos pueblos, con el objetivo de paliar un olvido demográfico, económico, social y cultural, se aferran a poner en valor sus tradiciones y su patrimonio (Knudsen y Wade; 2010; Cànoves y Blanco, 2011); asumiendo que si se pierde la memoria histórica también se acaba perdiendo la identidad (Cànoves y Prat, 2016). Es un hecho creciente el interés por la revalorización turística del patrimonio religioso material e inmaterial y ello se presenta como una oportunidad para la dinamización y afianzamiento de las identidades y tradiciones (Fernández Poncela, 2010; Capel, 2014; Cànoves y Prat, 2016).

En este contexto, el turismo cultural se presenta como uno de los principales productos turísticos al poner en valor el patrimonio histórico de un territorio. Asimismo, este tipo de turismo también ha servido para situar en el mapa turístico muchos lugares con dificultades para hacerse un hueco en un mercado cada vez más competitivo y segmentado (Cànoves y Blanco, 2011; Coma, 2014; Cors, 2014; López Palomeque y Font, 2014; Cànoves y Prat, 2016; Llurdés, 2016). De esta forma, algunos lugares religiosos han acabado transformándose en un elemento multifuncional y polifacético, 
punto de encuentro de turistas y de la población local (Esteve, 2002; Cànoves y Blanco, 2011). Además, los turistas, más experimentados, críticos, exigentes e individualistas, han modificado sus motivaciones e inquietudes, demandando unas actividades cada vez más personalizadas, auténticas y que les aporten valores y experiencias (Cànoves et al., 2012; Donaire, 2012; Prat, 2013).

En estos últimos años asistimos desde la geografía y otras disciplinas afines se han realizado interesantes aportaciones sobre esta temática. Entre otros autores, destacan los trabajos de Morinis, 1983; Esteve, 2002; Tilson, 2005; Andrés y Espejo, 2006; Brace et al., 2006; Buttimer, 2006; Cànoves, 2006; Ferber, 2006; Ivakhiv, 2006; Blackwell, 2007; Collins-Kreiner, 2010; Cànoves y Blanco, 2011; Lois y López, 2012; Millan et al., 2012; Shinde, 2012; Maddrell y della Dora, 2013; Olsen, 2013; Wilson et al., 2013; Cànoves et al., 2014; Cebrián y García, 2014; Cors, 2014; López Palomeque y Font, 2014; Pardellas y Padin, 2014; Uriely et al., 2015; Cànoves y Prat, 2016; Llurdés, 2016.

Por otra parte, también se está modificando la actitud de los residentes hacia los turistas, teniendo en cuenta, que una actitud es "un estado mental de disposición para responder que ejerce una influencia directa sobre la conducta" (Royo y Ruiz, 2009). Entre los factores más influyentes en las actitudes de los residentes hacia el turismo, se encuentran fundamentalmente dos: el tipo y grado de interacción entre los residentes y visitantes y la importancia de la actividad turística en el territorio (Murphy, 1983).

La teoría del intercambio social explica que el ser humano selecciona sus transacciones relacionales una vez ha evaluado sus beneficios y costes (Homans, 1961; Royo y Ruiz, 2009). Según esta idea, las actitudes se ven afectadas por las percepciones del intercambio que los individuos consideran que están realizando. De este modo, quienes se benefician más directamente del turismo son los que perciben que esta actividad genera mayores beneficios y menores impactos negativos (Getz, 1994).

En este contexto, un ejemplo de nuevas actividades que se están desarrollando alrededor del turismo cultural-religioso son las romerías. Se trata de excursiones grupales, generalmente a pié, a ermitas y/o santuarios situados en medio del campo o en las montañas, donde, además de las actividades puramente religiosas (misa, bendición pan, del territorio y de los feligreses), también se aprovecha para realizar otros actos festivos vinculados con las tradiciones del lugar (bailes, cantos populares, comidas comunitarias, etc.). En la actualidad, estos eventos históricos, lejos de desaparecer, están proliferando, dentro del auge que en estos últimos años está teniendo la demanda de turismo cultural, no exclusiva de las personas religiosas (Cors, 2014; Llurdés, 2016). Este incremento está muy relacionado con el deseo de los nuevos turistas de dar sentido a su tiempo de recreación y ocio (Cànoves et al., 2012; Coma, 2014).

Por ello, con las romerías, algunos lugares religiosos y más o menos emblemáticos se han convertido en puntos de encuentro de turistas, principalmente veraneantes con segundas residencias en el lugar o en sus alrededores, y de la población local, todos con diferentes motivaciones, la mayoría bastante alejadas de un sentimiento puramente religioso. Se trata de unas actividades festivo-turísticas basadas en eventos religiosos históricos que se han reconvertido en una atracción turística y en una herramienta de expansión de la cultura. Sin embargo, también son actividades de encuentro y de interrelación entre la población local y los no residentes habituales. 
Así, el presente trabajo tiene como principal objetivo analizar como las antiguas romerías se han convertido en un elemento más de atracción turística en los territorios de interior, con una triple función: revalorizar el patrimonio local, atraer gente y ser lugar de encuentro entre la comunidad local y los visitantes.

El estudio se ha llevado a cabo, a partir de la recogida de documentación sobre el tema y de una serie de entrevistas a participantes en este tipo de eventos. En primer lugar se analiza el caso de las romerías que anualmente se celebran en la comarca donde el turismo y las segundas residencias se han erigido como principales motores económicos. En segundo lugar, se presentar un breve resumen sobre la revalorización de las romerías como atractivo turístico y del actual grado de desarrollo turístico de la comarca ceretana, se analiza más detalladamente el caso de las romerías en la Cerdanya española (los llamados "aplecs"), a partir de una serie de datos estadísticos y un análisis cualitativo basado en cortas entrevistas a varias personas que han realizado esta actividad en la comarca en estos últimos años.

Consideramos que la comarca de la Cerdanya, en el Pirineo catalán, es un ejemplo de cómo se valoriza turísticamente el patrimonio religioso y las antiguas actividades folklóricas. Estas actividades revitalizan y conservan un patrimonio, muchas veces en vías de abandono, preservándolo del olvido y satisfaciendo las nuevas demandas culturales de la población (Cànoves et al. 2014; Donaire, 2012; Llurdés, 2016).

\section{LA VALORIZACIÓN TURÍSTICA DE LAS ROMERÍAS}

El diccionario de la Real Academia Española presenta tres definiciones de "romería":

1) Viaje o peregrinación, especialmente la que se hace por devoción a un santuario;

2) Fiesta popular que con meriendas, bailes, etc., se celebra en el campo inmediato a alguna ermita o santuario el día de la festividad religiosa del lugar;

3) Gran número de gentes que afluye a un sitio.

Para nuestro caso de estudio todas las definiciones son válidas y pueden complementarse entre ellas. En efecto, actualmente las romerías son unas peregrinaciones, con más o menos participantes, a un lugar sagrado, generalmente una iglesia, donde además de los actos puramente religiosos (misa, bendiciones) también se aprovecha para convertirlas en un encuentro festivo de carácter popular y folklórico (con reparto de pan bendito, coca y vino dulce, música, bailes, cantos, comidas y meriendas, etc.).

De esta forma, las romerías son un espacio de encuentro entre la comunidad local y los residentes estacionales, que generalmente tienen una segunda residencia en la población, lo que facilita su contacto y ayuda a limar posibles asperezas entre ambos grupos, que presentan distintas motivaciones, ya que la población local, aunque percibe los beneficios que aporta el turismo, incluyendo las segundas residencias, al territorio (más trabajo, aumento de las ventas en los comercios, cafeterías y restaurantes, mayor ocupación de los establecimientos turísticos, más ingresos municipales por licencias de obras e impuestos de bienes inmuebles, nuevos negocios, incremento de las actividades deportivas, lúdicas y recreativas, mejora de los servicios e infraestructuras públicas, etc.) también puede 
presentar algunos problemas (conflictos sociales y/o culturales ente ambos colectivos, problemas medioambientales, exceso de visitantes, urbanización desmedida, teatralización del territorio, creación de una ciudad dispersa, etc.) (Royo y Ruiz, 2009).

El turismo tiene un efecto instigador de cambio social (Harrison, 1992), ya que con esta actividad y el contacto entre turistas y residentes se potencia el orgullo de pertenencia a un lugar y la identidad cultural, se crean oportunidades de intercambio social y cultural y se dan a conocer y se restauran tanto el patrimonio como las tradiciones locales. Sin embargo, también puede causar una cierta artificialización y comercialización cultural desencadenando conflictos sociales en la comunidad de destino, debido a las diferencias socioculturales, de bienestar económico y de poder adquisitivo entre los residentes y los visitantes (Tosun 2002; Royo y Ruiz, 2009).

No se debe olvidar que el patrimonio cultural de un lugar está formado por un conjunto de elementos y manifestaciones producidas históricamente por las sociedades como resultado de un proceso evolutivo, donde la reproducción de las ideas y del material son factores que identifican y diferencian ese territorio de los demás (Prat, 2013). Dicho patrimonio incluye los elementos arquitectónicos del pasado (sitios y objetos arqueológicos) y las diversas manifestaciones de la cultura allí presentes, su artesanía y arte, su indumentaria, sus conocimientos, sus valores, su religión, sus costumbres y sus tradiciones. De esta manera, las romerías, ayudan a la recuperación y mantenimiento de los recursos patrimoniales religiosos y populares de un lugar y contribuyen a su revalorización como producto turístico. De este modo, patrimonio y turismo son dos elementos interdependientes que, además de favorecer el desarrollo local y la conservación patrimonial, facilitan los intercambios culturales y sociales en la comunidad.

Por ello, desde los ayuntamientos y las restantes instituciones públicas, sabedores de la importancia económica, social y cultural del turismo promueven este tipo de eventos. A partir de los eventos, los municipios pugnan por situarse en el mapa turístico de la comarca y favoreciendo el conocimiento de su patrimonio y facilitando la convivencia entre los residentes permanentes y temporales.

\section{EL DESARROLLO TURÍSTICO DE LA CERDANYA}

La comarca de la Cerdanya está situada (figuras 1 y 2) en el norte de Catalunya, en los Pirineos, limitando con Andorra, las comarcas francesas del Capcir, l'Arièja y el Conflent, y las españolas del Ripollès, el Berguedà y l'Alt Urgell. Es una de las comarcas naturales con una configuración más definida geográficamente (Llac, 1991; Blanchon et al., 2004; Prat, 2012, 2013), ya que está formada por un gran valle de hundimiento tectónico en dirección NE-NW, que va desde el Coll de la Perxa hasta el estrecho del Baridà, y está cruzada longitudinalmente por el río Segre.

Políticamente, como consecuencia del Tratado de los Pirineos, que puso fin a la Guerra entre Francia y España (1635-1659), la zona más oriental de la comarca pasó a Francia. A su vez, la parte española volvió a dividirse en 1833 entre las provincias de Lleida y Girona. Sin embargo, las relaciones familiares, socioeconómicas y culturales se han mantenido, siendo Puigcerdà la verdadera capital funcional de toda la comarca (Sahlins, 1991; Prat, 2012, 2013). 
Figura 1

\section{MAPA DE SITUACIÓN DE LA CERDANYA EN EUROPA}

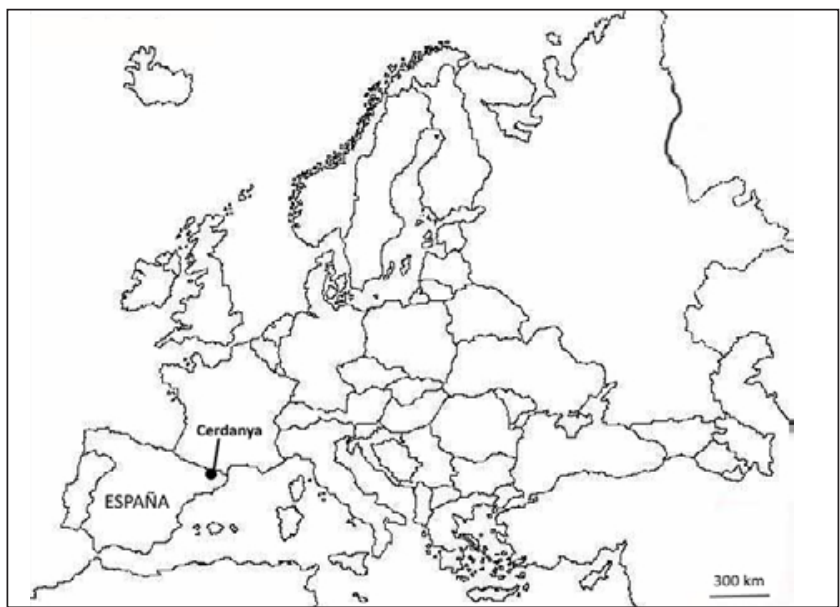

Fuente: elaboración propia.

Figura 2

MAPA DE SITUACIÓN EN CATALUNYA DE LA PARTE ESPAÑOLA DE LA CERDANYA

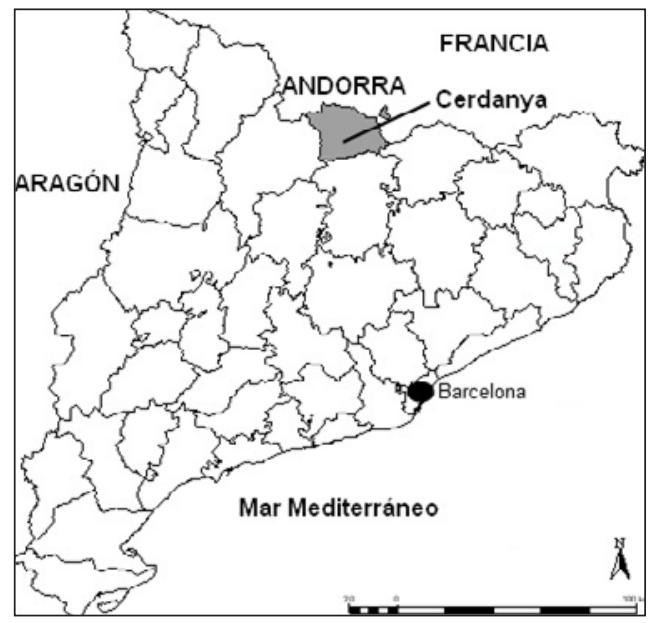

Fuente: elaboración propia con Miramon.

Sin embargo, la comarca, que ha sido históricamente agraria, ha modificado sustancialmente su actividad económica en estas últimas décadas, debido a su privilegiada situación geográfica, su paisaje y su climatología, y a una mejora sustancial de las comunicaciones 
terrestres con Barcelona y su Región Metropolitana. Así, la apertura del Túnel del Cadí en 1984 ha reducido significativamente el tiempo de desplazamiento entre la comarca y la capital catalana, ya que en la actualidad se puede realizar este trayecto en algo más de 1,5 horas, cuando anteriormente el viaje suponía algo más de 3 horas por la carretera de la Collada de Toses.

Esta facilidad de acceso, junto con la ampliación y mejora de los dominios esquiables, el mayor poder adquisitivo de la población, la consolidación de las vacaciones de verano y de invierno y el aumento del tiempo libre para el ocio y la recreación, ha propiciado que algunos barceloneses con un poder adquisitivo medio-alto y alto dispusieran de una segunda vivienda en la comarca. O simplemente pernoctasen algunas temporadas a lo largo del año (especialmente durante los periodos vacacionales de Navidad, Semana Santa, los puentes festivos y las vacaciones de verano) en los múltiples establecimientos hoteleros, casas rurales y cámpings de la comarca.

Así, según datos de 2016 del Institut d'Estadística de Catalunya (Idescat), la oferta de plazas hoteleras en la comarca ha ido creciendo paulatinamente en estos últimos años, pasado de 2.506 en el 2003 (de las que 1.726 eran en hoteles y 780 en hostales y pensiones) a 3.007 en el 2015 ( 2.568 en hoteles y 439 en hostales y pensiones), lo que representa un aumento del 19,99\% en este período (2003-2015), destacando el incremento del 48,78\% en la oferta de plazas en los hoteles y la disminución del 43,72\% en los hostales y pensiones. Análogamente, las plazas de turismo rural en la comarca a lo largo del período 2003-2015 han aumentado un $31,85 \%$, pasando de 292 a 385 . En cuanto a los cámpings, se ha pasado de 3.771 plazas en el año 2003 a 4.524 plazas en el 2015, lo que representa un aumento del 19,96\%. Desde la demanda, el grado de ocupación de los establecimientos turísticos de la comarca es muy estacional, dependiendo de la temporada, superándose solamente una ocupación del $65 \%$ durante las dos semanas intermedias del mes de agosto, Semana Santa y el puente de la Constitución.

Sin embargo, donde mejor se aprecia la importancia turística de este destino es en las segundas residencias, ya que de las 21.186 viviendas censadas en la comarca en el año 2011, solamente 7.665 eran principales (el 36,18\% del total), mientras que de las 13.521 restantes, 11.353 eran secundarias (el $53,58 \%$ ) y 2.168 se declaraban como vacías (el 10,24\%). Además, hay que tener en cuenta que 9.453 de las segundas residencias fueron construidas a partir de 1971 (el 81,95\% de las existentes) y de ellas, más de la mitad (el 56,10\%) se edificaron a partir de 1991. (Idescat, 2016). Así pues, la comarca tiene un parque de viviendas relativamente moderno, ya que el inicio del "boom" de la construcción, especialmente con las segundas residencias, se produjo unos pocos años antes de la inauguración del Túnel del Cadí, cuando la población urbana, fundamentalmente barcelonesa, empezó a apostar por este destino como segunda residencia, tanto en verano como en invierno, consolidándose la comarca como un referente de turismo de interior de alta calidad.

\section{LAS ROMERÍAS EN LA CERDANYA}

La comarca de la Cerdanya atesora más de 60 iglesias con vestigios románicos diseminadas por todo el valle (Ventosa, 2004). Este rico patrimonio, ha facilitado las romerías como encuentros religiosos y lúdico-festivos, donde la peregrinación y la misa iban acom- 
pañados de la bendición de las tierras. Recientemente, los municipios han aprovechado estos tradicionales eventos como reclamo turístico y revalorización patrimonial, en donde a partir de las actividades tradicionales se fomenta el encuentro entre la comunidad local y los visitantes, a partir de comidas o meriendas populares, música tradicional, bailes, cantos, juegos infantiles, etc.

Así, en la actualidad, en la parte española de la comarca se realizan diez romerías (allí denominados "aplecs"). Son los "aplecs" de la Font de Talló (en el término municipal de Bellver), Quadres (en Isòvol), Bastanist (en Martinet i Montellà), Sant Salvador de Predanies (en Prats i Sansor), Sant Marc y Rigolisa (ambas en Puigcerdà), Fontanera (en Guils), Vilagrau (en Urús), el Remei (en Bolvir) y la Xicola (en Meranges). Asimismo, hay otros dos en la parte francesa, los de Bell-lloch (en Dorres) y Font-Romeu.

\section{Figura 3 \\ MAPA DE SITUACIÓN DE LAS ROMERÍAS EN LA CEDANYA ESPAÑOLA}

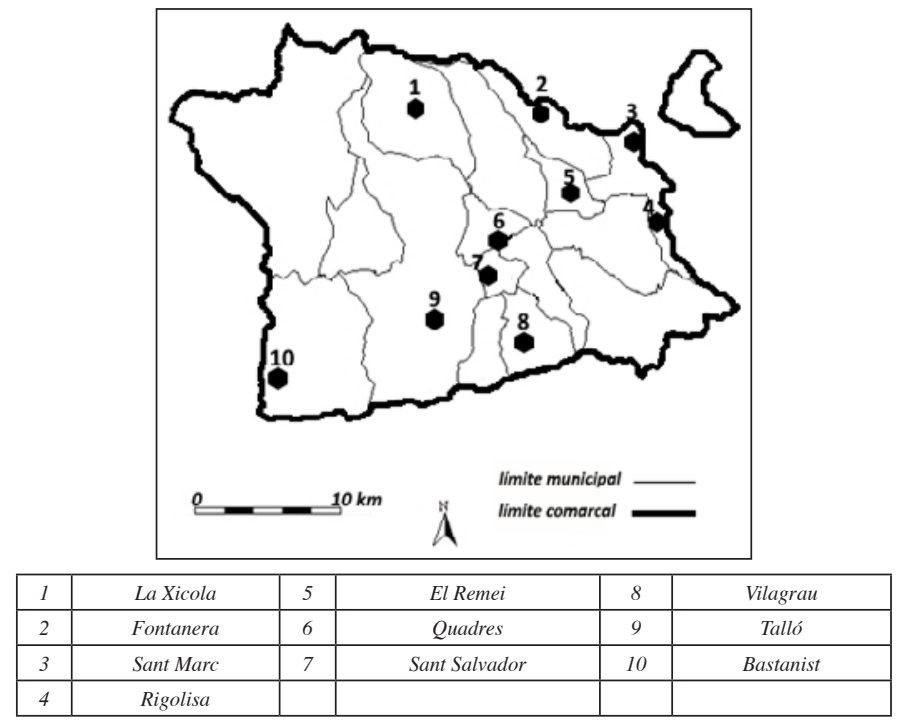

Fuente: elaboración propia con Miramon.

Una de las romerías más antiguas y populares que se celebran en la parte española de la comarca es la de la Font de Talló, detrás del montículo de Monterrós, cuando en primavera se peregrina hasta la fuente y se reza a la Virgen situada en una capillita adjunta con el propósito de favorecer el resurgir de la naturaleza. Antiguamente, se aprovechaba dicho evento como lugar de encuentro de los jóvenes de la comarca, lo que facilitaba muchos noviazgos. Ahora, por la mañana, en la iglesia parroquial de Bellver se celebra una misa, se cantan caramellas (canciones populares típicas de Catalunya para celebrar la Resurrección de Jesús) y se bailan sardanas. Por la tarde, se peregrina hasta la fuente, se vuelven a cantar caramellas y bailar sardanas y se reparte pastel y vino dulce. Finalmente, por la noche en el pueblo se celebra un baile. 
Otra romería importante es la que se celebra cada 6 de agosto en la ermita románica (siglo XI) de Sant Salvador de Predanies, en la cumbre de un montículo que domina parte del valle. En ella, por la mañana, se peregrina hasta la ermita, en cuyos alrededores se celebra una misa, se bendice la comarca y se reparte pan bendito y vino dulce. Luego, por la tarde, en el pueblo de Prats se celebran juegos infantiles y se reparte coca y chocolate. También destaca la romería de Bastanist, cuando cada primer domingo de mayo se peregrina hasta el Santuario de la Virgen negra de Bastanist, para celebrar el hallazgo de la cruz de Jesús por parte de Santa Elena. Allí se celebra una misa, se bendicen los municipios de los alrededores, se celebra una comida popular y se realizan cantos religiosos en honor a la Virgen María.

Las restantes romerías que se celebran anualmente son la de Sant Marc, el 25 de marzo, con una caminata y una misa en la ermita en honor de este santo. La del Remei, cuando por Sant Joan se celebra una misa y se bailan sardanas en la ermita de Nuestra Señora del Remei, adosada al actual hotel de la Torre del Remei. La de la Xicola, en Meranges, cada primer domingo de junio. La de Fontanera, en la estación de esquí nórdico de GuilsFontanera, en el mes de agosto, con una excursión a pie desde Guils, una misa de campaña, una comida popular y juegos infantiles. La de Vilagrau, en noviembre, en Urús, con una excursión a la ermita de Sant Grau. La de Quadres, en el segundo domingo de septiembre, con una misa, bailes de sardanas y una comida popular en el renovado santuario de Santa María de Cuadres, que presenta vestigios del siglo XII y antigua hospedería medieval junto al camino real de la Cerdanya. La de Rigolisa, cada 25 de julio, con una merienda y bailes de sardanas al lado de una pequeña ermita junto a la actual masía del mismo nombre, antes un pequeño pueblo cercano a Puigcerdà y documentado desde el siglo $\mathrm{X}$.

En el cuadro siguiente (tabla 1) se presenta una relación de las diez romerías que actualmente se celebran en la parte española de la comarca, así como las actividades principales que se realizan en cada una de ellas.

Tabla 1

LISTA DE LAS ROMERÍAS Y SUS ACTIVIDADES EN LA CERDANYA ESPAÑOLA

\begin{tabular}{|c|c|c|c|c|c|c|}
\hline & & Caminata & Misa & $\begin{array}{c}\text { Sardanas/Bailes/ } \\
\text { Canciones }\end{array}$ & $\begin{array}{l}\text { Comida/ } \\
\text { Merienda }\end{array}$ & $\begin{array}{c}\text { Juegos } \\
\text { infantiles }\end{array}$ \\
\hline 1 & La Xicola & $\mathrm{x}$ & & & & \\
\hline 2 & Fontanera & $\mathrm{x}$ & $\mathrm{x}$ & & $\mathrm{x}$ & $\mathrm{x}$ \\
\hline 3 & Rigolisa & $\mathrm{x}$ & & $\mathrm{x}$ & $\mathrm{x}$ & \\
\hline 4 & Sant Marc & $\mathrm{x}$ & $\mathrm{x}$ & & & \\
\hline 5 & El Remei & & $\mathrm{x}$ & $\mathrm{x}$ & & \\
\hline 6 & Quadres & & $\mathrm{x}$ & $\mathrm{x}$ & $\mathrm{x}$ & \\
\hline 7 & Sant Salvador & $\mathrm{x}$ & $\mathrm{x}$ & & $\mathrm{x}$ & $\mathrm{x}$ \\
\hline 8 & Vilagrau & $\mathrm{x}$ & & & & \\
\hline 9 & Font de Talló & $\mathrm{x}$ & $\mathrm{x}$ & $\mathrm{x}$ & $\mathrm{x}$ & \\
\hline 10 & Bastanist & & $\mathrm{x}$ & $\mathrm{x}$ & $\mathrm{x}$ & \\
\hline
\end{tabular}


Asimismo, en la parte francesa de la comarca ceretana, destacan las romerías de Bell-lloch y Font-Romeu. La primera, se compone de una excursión a pié en septiembre desde Dorres al santuario de Santa María de Bell-lloch, que presenta algunos vestigios del siglo XIII, y allí se realizan varias actividades, como un desayuno, una misa, una comida popular y bailes de sardanas. La segunda, más formal y multitudinaria (con más de 2.000 asistentes), cada primer domingo de septiembre en el santuario de la Virgen de Font-Romeu, con una misa concelebrada y bailes de sardanas.

El impacto de estos eventos lúdico-religiosos se centra en dos aspectos. Por una parte, la recuperación y/o mantenimiento de un patrimonio y de unas tradiciones. Por otro, constituir un punto de encuentro social de la comunidad local y los visitantes. La tabla 2 refleja los comentarios más significativos de los 14 entrevistados, teniendo en cuenta el perfil de los entrevistados.

Tabla 2

PERFIL DE LOS ENTREVISTADOS

\begin{tabular}{|l|c|c|c|}
\hline Total entrevistados & & 14 & $100 \%$ \\
\hline \multirow{2}{*}{ Género } & Masculino & 6 & $42,86 \%$ \\
\cline { 2 - 4 } & Femenino & 8 & $57,14 \%$ \\
\hline \multirow{2}{*}{ Edad } & Hasta 60 años & 10 & $71,43 \%$ \\
\cline { 2 - 4 } & Más de 60 años & 4 & $28,57 \%$ \\
\hline \multirow{2}{*}{ Lugar de residencia habitual } & En la misma población & 5 & $35,71 \%$ \\
\cline { 2 - 4 } Tipo de residencia en la comarca & En otra población & 9 & $64,29 \%$ \\
\hline \multirow{2}{*}{ Veces que han realizado la romería } & Residencia habitual & 6 & $42,86 \%$ \\
\cline { 2 - 4 } & Residencia temporal & 8 & $57,14 \%$ \\
\cline { 2 - 4 } & 5 o menos & 3 & $21,43 \%$ \\
\hline
\end{tabular}

C1: "Es el momento en que puede considerarse que empieza verdaderamente el verano. Allí nos reunimos muchos del pueblo y los veraneantes. Nos vemos, comentamos como ha ido el año. En fin, nos ponemos al día"

C2: "Es una fiesta muy tradicional. Ya hace tiempo que la hacemos. Después de la misa, mientras comemos la coca, hablamos todos, nos saludamos con los del pueblo y comentamos nuestras cosas. Lástima que muchas veces hace un calor de mil demonios, pero a mí me gusta mucho ir”

C3: "Es una tradición muy bonita. Ha servido para que mucha gente conozca esta ermita. Además nos vemos todos. Yo antes iba andando, pero ahora que ya soy mayor me llevan en coche, pero si puedo no me la pierdo"

C4: "Allí nos volvemos a encontrar muchos al cabo de un año. Me gusta ir con mis nietos. (...) Saludamos a unos y otros. Bailamos sardanas. Es una fiesta muy familiar” C5: "Es una fiesta muy tradicional, aunque cada vez viene más gente de fuera que nunca he visto. Algunos ni son del pueblo" 
C6: "El vino que dan cada año está muy bueno. Hablo con todos y luego vuelvo a casa muy animada"

C7: "Cada año recojo pan bendito para toda la familia y para los vecinos que no han podido ir. El bollo me lo guardo en casa todo el año. Es una tradición”

C8: "La vista es incomparable. Desde allí se divisa casi toda la Cerdanya. Como que somos muchos y no cabemos todos en la iglesia, la misa se hace al aire libre y pasamos mucho calor. Pero a mí me gusta ir, nos dan coca, hablamos unos con otros. En fin, vemos a mucha gente que no habíamos visto en mucho tiempo"

C9: "Es una tradición muy bonita. No podemos dejarla perder. Cada verano voy. Así puedo ver a mucha gente del pueblo y hablamos. Si no, ni los vería nunca. (...) La misa es lo de menos. Lo bonito es la fiesta que hay después. Las sardanas, la música. Todo"

C10: "Comemos un arroz muy bueno. Y hablamos unos con otros. Es muy agradable. Veo a gente que casi nunca aparecen por el pueblo"

C11: “A veces no puedo ir, pero si puedo voy. Me acompañan algunas vecinas. Escuchamos misa y comemos coca. Hablamos con gente que hacía tiempo que no veíamos. Me gusta ir"

C12: "Escuchamos misa, comemos, hablamos. En fin, es un día muy agradable. Vale la pena ir, aunque a veces estoy fuera y no puedo ir. Es una lástima. (...) Es un poco como volver a la escuela después de las vacaciones. Te reencuentras con los amigos. Hablas con todo el mundo. Te pones al día de todo"

C13: "Al ir andando hasta la ermita, miro los campos y el paisaje, y me impregno cada año de una sensación muy agradable. Vuelvo a mi casa y veo a mi gente. (...) No soy religioso pero me gusta ir a esta misa. Es una tradición muy bonita" C14: "Con estas fiestas mantenemos las tradiciones de aquí, que habían sido olvidados durante muchos años. Hemos de hacer un esfuerzo para que los jóvenes también participen y no las dejen de lado. Ellos son el futuro de nuestro pueblo"

Como puede apreciarse en las anteriores respuestas, entre los participantes en las romerías predomina el aspecto lúdico-festivo sobre el puramente religioso. Destacan especialmente las relaciones sociales, ya que estos eventos han sido históricamente y siguen siendo en la actualidad un apropiado lugar de encuentro de gentes que viven habitualmente en el pueblo y otras que no, lo que les permite saludarse, hablar y ponerse al día de las noticias de cada uno. Este fenómeno es más destacable en aquellos y aquellas que aún residiendo la mayor parte del año fuera, además de poseer allí una segunda residencia, mantienen vínculos familiares con el pueblo. Las fiestas tradicionales han tenido en los espacios rurales de Catalunya un crecimiento considerable en los últimos años, en versiones variadas como son los aplecs, romerías, encuentros corales, fiestas de la vendimia, celebraciones religiosas, o los más habituales festivales medievales. Estas actividades tal como expresan los entrevistados, reflejan por un lado el sentimiento de pertenencia al lugar y la comunidad, la valorización del patrimonio tangible e intangible, el arraigo a las tradiciones y sobretodo la reunión de locales y visitantes. 
El caso de la Cerdanya, es emblemático en Cataluña, ya que junto con la importancia de la segunda residencia y por tanto la existencia de una notable comunidad de personas residentes temporalmente, se unen los visitantes y los locales. Las celebraciones han ido incrementándose en base a la demanda de los residentes habituales y a la presión de los residentes temporales que sienten más propio su lugar de residencia a medida que se pueden sentir más integrados en la comunidad local. Esta dinámica favorece el turismo, que acoge de buen grado actividades vinculadas a tradiciones culturales que se han perdido en los espacios urbanos de donde provienen la mayor parte de los asistentes.

\section{CONCLUSIONES}

En estas últimas décadas se han producido importantes cambios en el sector turístico, con nuevas motivaciones, expectativas e intereses de los turistas y con una nueva oferta de productos, cada vez más temáticos y específicos; así como también en las relaciones sociales generadas alrededor el turismo. Ello ha facilitado el auge del turismo cultural en los territorios de interior, cuyo consumo provoca una satisfacción a la que no es ajena la imagen proyectada, tanto en la comunidad local como en los visitantes, ayudando a consolidar el destino como foco de atracción del patrimonio allí existente (Cànoves, et al., 2014; Cànoves y Prat, 2016).

En este artículo se ha confirmado como el factor emocional es relevante en la experiencia de consumo del turismo cultural-religioso, ya que las emociones son el elemento central en la satisfacción del turista y, como consecuencia, la gestión de sus expectativas debe completarse con la gestión de dichas emociones. Por ello, los destinos turísticos de interior, aprovechando sus recursos patrimoniales y el impulso de la demanda existente, deben ser capaces de ofrecer una diversidad de actividades complementarias que permitan experimentar a los participantes unas emociones de distinta intensidad, pero que les satisfagan y les comprometan emocionalmente con el lugar, creando un vínculo y diferenciándolo de los demás.

Así, en el caso de las romerías de la Cerdanya, nos encontramos ante una actividad cultural y/o religiosa que deriva en un efecto positivo para la comarca, ya que, aprovechando su poder de atracción, también contribuye a la revalorización, restauración, preservación y protección, de unos recursos culturales algunas veces olvidados. A partir de dicha celebración se contribuye a preservar la identidad cultural del territorio y a facilitar las relaciones sociales entre la comunidad local y los turistas, incluyendo los residentes temporales.

En este trabajo, a partir de los resultados obtenidos en la comarca de la Cerdanya, se puede constatar que a través de eventos culturales lúdico-religiosos se establece una relación positiva entre la comunidad local y los turistas, reafirmando el destino como un punto de encuentro de ambos colectivos y presentándolo, además, como lugar de interés turístico por su valor patrimonial.

Así pues, las romerías son un ejemplo de actividades religioso-culturales cuya revaloración turística ayuda a reafirmar la identidad local, conservar el patrimonio material e inmaterial y, además, generar unas relaciones sociales entre unos segmentos de población con diferentes intereses y motivaciones. 
Finalmente, los autores del artículo son conscientes de que la muestra de entrevistas es reducida, por lo que en el futuro debemos aumentarla y profundizar en el conocimiento de nuevos elementos que permitan medir y contrastar el apoyo de la población local al desarrollo turístico de unos territorios de interior muy diferentes, lo que ayudará a ampliar el conocimiento de los determinantes de la actitud de esta población hacia el turismo y el visitante, y así desde las administraciones públicas, la sociedad civil y las empresas, estar en condiciones de diseñar una oferta sostenible y cada vez más acorde con las nuevas demandas de los turistas y con los intereses locales, demasiado olvidados en estos últimos años de un "boom" turístico indiscriminado.

\section{BIBLIOGRAFÍA}

ANDRÉS, J.L. y ESPEJO, C. (2006): «Interacción mito religioso / producto turístico en la imagen de la ciudad: Caravaca de la Cruz (Murcia)». Cuadernos de Turismo, nº 18, pp. 7-61.

BLACKWELL, R. (2007): «Motivations for religious tourism, pilgrimage, festivals and events», en Raj, R. y Morpeth, N.D. (Eds.). Religious tourism and pilgrimage festivals management. An international perspective. Cambridge: Cab International, pp. 35-47.

BLANCHON, J.L.; BOSOM, S.; MERCADAL, O. y SOLER, M. (2004): La guía de tota la Cerdanya i el Capcir. Barcelona, Editorial Sinopsis.

BRACE, C., BAILEY, A.R., y HARVEY, D.C. (2006): «Religion, place and space: A framework for investigating historical geographies of religious identities and communities». Progress in Human Geography, no 30 (1), pp. 28-43.

BUTTIMER, A. (2006): «Afterword: Reflections on Geography, religion, and Belief Systems». Annals of the Association of American Geographers, $\mathrm{n}^{\circ} 96$ (1), pp. 197-202.

CÀNOVES, G. (2006): «Turismo religioso en Montserrat: montaña de fe, montaña de turismo». Cuadernos de Turismo, $\mathrm{n}^{\circ}$ 18, pp. 63-76.

CÀNOVES, G. y BLANCO, A. (2011): «Turismo religioso en España: ¿la gallina de los huevos de oro? Una vieja tradición, versus un turismo emergente». Cuadernos de Turismo, $\mathrm{n}^{\mathrm{o}} 27$, pp. 115-131.

CÀNOVES, G.; ROMAGOSA, F.; BLANCO, A. y PRIESTLEY, G. (2012): «Religious tourism and sacred places in Spain: old practices, new forms of tourism». International Journal of Tourism Anthropology, no 2 (4), pp. 282-298.

CÀNOVES, G.; VILLARINO, M.; BLANCO, A.; DE UÑA, E. y ESPEJO, C. (Eds.) (2014): Turismo de interior: renovarse o morir. Estrategias y productos en Catalunya, Galicia y Murcia. Valencia, Publicacions de la Universitat de València.

CANOVES, G. y PRAT J.M. (2016): «The Determinants of Tourist Satisfaction in Religious Destinations: the case of Montserrat (Spain)». International Journal of Religious Tourism and Pilgrimage, $\mathrm{n}^{\circ} 4$ (5), pp. 26-36.

(Disponible en: http://arrow.dit.ie/ijrtp/vol4/iss5/5) (Consultado el 26 de octubre de 2016)

CAPEL, H. (2014): El patrimonio: la construcción del pasado y del futuro. Barcelona, Ediciones del Serbal.

CEBRIÁN, A. y GARCÍA, R. (2014): «Del turismo religioso a las peregrinaciones permanentes: diversificación turística en el sureste español». Cultur, Revista de Cultura e Turismo, $\mathrm{n}^{\mathrm{o}} 2$, pp. 3-30. 
COLLINS-KREINER, N. (2010): «The geography of pilgrimage and tourism: transformations and applications for applied geography». Applied Geography, $\mathrm{n}^{\circ} 30$ (1), pp. 153-164.

COMA, J. (2014): «Nuevos lugares y productos turísticos en la Cataluña interior. Los productos que no aparecen en el mapa de destinos turísticos catalanes», en LÓPEZ PALOMEQUE, F. y CÀNOVES, G. (eds.). Turismo y territorio. Innovación, renovación y desafíos. Valencia, Tirant Humanidades, pp. 415-428.

CORS, M. (2014): «La proyección turística como estrategia de desarrollo económico y renovación urbana en ciudades medias de interior. El caso de Manresa (Cataluña) » (póster), en LÓPEZ PALOMEQUE, F. y CÀNOVES, G. (Eds.). Turismo y territorio. Innovación, renovación y desafíos. Valencia, Tirant Humanidades, p. 595.

DONAIRE, J.A. (2012): Turismo cultural. Entre la experiencia y el ritual. Girona, Editorial Vitel·la.

ESTEVE, R. (2002): Turismo y religión. Aproximación a la historia del turismo religioso. Málaga, Servicio de publicaciones de la Universidad de Málaga.

FERBER, M. (2006): «Critical realism and religion: objectivity and the insider/outsider problem». Annals of the Association of American Geographers, ${ }^{\circ} 96$ (1), pp. 176-181.

FERNÁNDEZ PONCELA, A.M. (2010): «El Santo niño de Atocha: patrimonio y turismo religioso». Pasos, Revista de Turismo y Patrimonio Cultural, $\mathrm{n}^{\circ} 8$ (2), pp. 375-387.

GETZ, D. (1994): «Residents's attitudes towards turism: A longitudinal study in Spey Valley, Scotland». Tourism Management, $\mathrm{n}^{\circ}$ 15, pp. 247-258.

HARRISON, D. (1992): Tourism to less developed countries: The social consequences in tourism and less developed countries. London, Bellhaven.

HOMANS, G. (1961): Social Behavior: Its Elementary Forms. New York, Harcourt Brace Jovanovich.

IVAKHIV, A. (2006): «Towards a geography of religion: mapping the distribution of an unstable signifier». Annals of the Association of American Geographers, $\mathrm{n}^{\circ} 96$ (1), pp.169-175.

KNUDSEN, B.T. y WADE, A.M. (2010): Re-Investing Authenticity, Tourism, Place and Emotions. Bristol, Channel View Publications.

LLAC, F. (1991): «Histoire géologique de la Cerdagne». Ceretania, nº 1, pp. 7-20.

LOIS, R.C. y LÓPEZ, L. (2012): «El Camino de Santiago: una aproximación a su carácter polisémico desde la geografía cultural y el turismo». Documents d'Anàlisi Geogràfica, $\mathrm{n}^{\circ} 58$ (3), pp. 459-479.

LÓPEZ PALOMEQUE, F. y FONT, X. (2014): «Evolución reciente de la gestión turística local en los espacios de interior de Cataluña», en LÓPEZ PALOMEQUE, F. y CÀNOVES, G. (eds.). Turismo y territorio. Innovación, renovación y desafíos. Valencia, Tirant Humanidades, pp. 479-490.

MADDRELL, A. y DELLA DORA, V. (2013): «Crossing surfaces in search of the holy: landscape and liminality in contemporary Christian pilgrimage». Environment and Planning, $\mathrm{n}^{\circ} 45$ (5), pp. 1105-1126.

MILLÁN, M.G., PÉREZ, L.M. y MARTÍNEZ, R. (2012): «Etapas del ciclo de vida en el desarrollo del turismo religioso: una comparación de estudios de caso». Cuadernos de Turismo, $\mathrm{n}^{\mathrm{o}}$ 30, pp. 241-266. 
MORINIS, A. (1983: «Pilgrimage and tourism». Annals of Tourism Research, $\mathrm{n}^{\circ} 10$ (4), pp. 569-570.

MURPHY, P.E. (1983): «Community Attitudes to Tourism». Tourism Management, $\mathrm{n}^{\circ} 2$, pp. 189-195.

OLSEN, D.H. (2013): «A scalar comparison of motivations and expectations of experience within the religious tourism market». International Journal of Religious Tourism and Pilgrimage, $\mathrm{n}^{\mathrm{o}} 1$ (1), pp. 41-61.

PARDELLAS, X. y PADÍN, C. (2014): «El Camino de Santiago portugués en Galicia y las estrategias turísticas locales». Revista de Análisis Turístico, $\mathrm{n}^{\circ}$ 17, pp. 51-59.

PRAT, J.M. (2012): «La població i les activitats econòmiques de la Cerdanya a l'últim quart del segle XVIII (1775-1800)». Treballs de la Societat Catalana de Geografia, $\mathrm{n}^{\mathrm{o}} 73$, pp. 167-192.

PRAT, J.M. (2013): «Evolució del turisme rural a la Baixa Cerdanya (200-2012)». Querol. Revista Cultural de Cerdanya, n ${ }^{\circ} 13$, pp.36-38.

REAL ACADEMIA ESPAÑOLA (2016): Diccionario de la lengua española. Madrid, RAE. (Disponible en: http://dle.rae.es/?id=WeRtrBl) (Consultado el 4 de noviembre de 2016).

ROYO, M. y RUIZ, M.E. (2009): «Actitud del residente hacia el turismo y el visitante: factores determinantes en el turismo y excursionismo rural-cultural». Cuadernos de Turismo, $\mathrm{n}^{\mathrm{o}} 23$, pp. 217-236.

SAHLINS, P. (1991): «Llenguatge, identitat i la Revolució francesa: visió de la perifèria». Ceretania, $\mathrm{n}^{\circ} 1$, pp. 135-149.

SHINDE, K. (2012): «Policy, planning, and management for religious tourism in Indian pilgrimage sites». Journal of Policy Research in Tourism, Leisure and Events, $\mathrm{n}^{\circ} 4$ (3), pp. 277-301.

TILSON, D.J. (2005): «Religious-spiritual tourism and promotional campaigning: A Church-state partnership for St. James and Spain». Journal of Hospitality \& Leisure Marketing, ${ }^{\circ} 12$ (1), pp. 9-40.

TOSUN, C. (2002): «Host perceptions of impacts: A comparative tourism study». Annals of Tourism Research, $\mathrm{n}^{\mathrm{o}} 29$, pp. 231-245.

URIELY, N., ISRAELI, A., y REICHEL, A. (2015): «Religious identity and residents' attitudes toward heritage tourism development: The case of Nazareth». Journal of Hospitality and Tourism Research, $\mathrm{n}^{\circ} 27$ (1), pp. 69-84.

VENTOSA, E. (2004): Les esglésies romàniques de la Cerdanya. Sant Vicenç de Castellet (Barcelona), Farell Editors.

WILSON, G.B., MCINTOSH, A.J., y ZAHRA, A.L. (2013): «Tourism and Spirituality: A phenomenological analysis». Annals of Tourism Research, $\mathrm{n}^{\circ} 42$, pp. 150-168. 
